\title{
Oxygen Specific Power Consumption Comparison for Air Separation Units
}

\author{
Yas A. Alsultanny ${ }^{*}$ and Nayef N. Al-Shammari \\ Arabian Gulf University, College of Graduate Studies, Kingdom of Bahrain \\ *E-mail: alsultannty@hotmail.com
}

\begin{abstract}
Technologies life cycles became shorter than before as a result of globalization and open market, which derived organizations to update their dated technologies. Without technologies updating, which based on Technological Forecasting (TF), organizations can not be dominant leaders in the open market and eventually they will lose their business. The main objective of this paper was to evaluate the air separation units by calculating the oxygen specific power, to find the most cost effective unit. The oxygen specific power used as a Key Performance Indicator (KPI) for the selected Air Separation Unit (ASU) technologies. The KPI for the updated Air Separation Unit was reviewed and the latest value selected as theoretical benchmark, which was $0.28 \mathrm{Kw} / \mathrm{Nm}^{3}$. At the practical part, the data collected to three air separation units ASU-31, ASU-51 and ASU-71. The results showed that the specific power gaps that used as the KPI's of ASU-31, ASU-51 and ASU-71 are $0.464 \mathrm{Kw} / \mathrm{Nm}^{3}, 0.639 \mathrm{Kw} / \mathrm{Nm}^{3}$ and $0.631 \mathrm{Kw} / \mathrm{Nm}^{3}$ respectively. The results showed that these gaps can be minimized by the recommendation suggested in this paper to reduce power consumption.
\end{abstract}

Keywords: Air separation unit, oxygen specific power, technological forecasting, key performance indicators.

ENGINEERING JOURNAL Volume 18 Issue 2

Received 3 February 2013

Accepted 1 August 2013

Published 18 April 2014

Online at http://www.engj.org/

DOI:10.4186/ej.2014.18.2.67 


\section{Introduction}

Technology Forecasting (TF) used for the purpose of future identification. Betz in 2003 [1] highlighted that technological change was finiteness process and TF can be used as methodology to trace the technological progress. Technology forecasting needs to be implemented effectively through all the life cycle of technologies to enhance the accuracy of the decisions making. Forecasting generally defined as a prediction of unknown situation in the future; it was widely used in production and demand forecasting, forecasting deals with the characteristics of a technology, like speed of a military aircraft, fuel consumption by cars, and performance of a machine with respect to operating cost and production capacity in coming years. Technological forecasting developed in 1970's to predict a technical achievement within a specified period at a given level of support within a given confidence level, this view was not valid any more with the following years of that period. The scope of technology forecasting was changing from time to time with respect to capability and accuracy [2].

The rate of technologies development was not the same, technology life cycle became shorter than before because of the huge developments, that were taking place in a short time. For example, Information Technology was one of the technologies that were having short life cycle. Similarly, technologies used in defense sector also have short life cycle. All the technologies used for production, had relatively longer life cycles than other technologies. It was argued that an organization can only be as dominant leaders in the market if they have capability to forecast and implement the development on the technologies being used in their businesses, it was argued that manufacturing organizations were transformed from producing organization to a learning organization $[3,4]$.

All chemicals and petrochemicals companies used industrial gases like oxygen and nitrogen either as a feedstock or as utilities, therefore this paper is concerning comparison between the technological forecasting for air separation units of oxygen and nitrogen gases to forecasting the methods of less power consumption and cost effective. The three units available to this research were ASU-31, ASU-51, and ASU71. Industrial gases processes classified into Non-Cryogenic Industrial Gas Process and Cryogenic Industrial Gas Process. The Non-Cryogenic Industrial gas processes operated at approximately ambient temperature and cannot produced oxygen and nitrogen in large scale; therefore in this paper Cryogenic Industrial Gas Process was considered, as the most efficient and cost effective process for production of large quantities of oxygen, nitrogen, and argon in gaseous or liquid forms, also with high purity.

\section{Classification of Technological Forecasting Methods}

Knowing the different TF methods and their capabilities enhances the proper selection of TF method and leads to an effective implementation for the selected TF. Armstrong in 1989 [5] and Slocum in 2001 [6] stated Technology Forecasting has evolved from being a methodology based on emotional responses to one predicated on data collection, moreover, the quantity and quality of available data required for TF are not the same for all the cases. The methodology of forecasting process in general classified in two main categories to judgmental methods and statistical methods, the judgmental methods are built on experience and expectation of people, where the statistical methods are built on data collection [7]. Mishra, et al in 2002 reviewed technological forecasting methods by classifying these methods to three categories [8]:

- Subjective assessment methods.

- Exploratory methods.

- Normative methods.

Porter, et al. In 2004 introduced an umbrella concept covering technological forecasting process with name of Technology Future Analysis (TFA), they classified the methods of TFA into two main categories: Qualitative which is built on empirical, numerical data and Quantitative as a Judgmental method based on knowledge [9].

Firat, et al. in 2008 stated that there are hundreds of TF methods that can be fit into 9 families; these are [10]:

- Expert Opinion.

- Trend Analysis.

- Monitoring and Intelligence Methods.

- Statistical Methods 
- Modeling and Simulation

- Scenarios.

- Valuing/Decision/Economics Methods

- Descriptive and Matrices Methods.

- Creativity.

According to the above, the suitable method of technology forecasting selected in this paper was quantitative method based on historical data mining and theoretical information as a benchmark. The model specification establishment based on the performance of selected ASUs, represented by a mathematical correlation specified as oxygen specific power $\left(\mathrm{Kw} / \mathrm{Nm}^{3}\right)$. This model was done to ensure that, the selected Key Performance Indicator (KPI) was representing the less power consumption and cost effective.

\section{Air Separation Units}

Fresh air is very essential for all living things; everybody can sense how air is important in his life. Air is not a single element but it is a mixture of chemicals in gases form, it may contain impurities like dust particles and water vapor as well as released gases known as pollutant. Table 1 shows the fresh air components and their percentage at the sea level [11].

Table 1. Fresh air components [11].

\begin{tabular}{lclc}
\hline Component & Fraction of air & Component & Fraction of air \\
\cline { 2 - 5 } Nitrogen & $78.09 \%$ & Methane & $1-2 \mathrm{vppm}$ \\
Oxygen & $20.95 \%$ & Acetylene & $<1 \mathrm{vppm}$ \\
Argon & $0.93 \%$ & Krypton & $1.14 \mathrm{vppm}$ \\
Carbon dioxide & $350 \mathrm{vppm}$ & Nitrous oxide & $0.5 \mathrm{vppm}$ \\
Carbon monoxide & $3-5 \mathrm{vppm}$ & Hydrogen & $0.5 \mathrm{vppm}$ \\
Neon & $18 \mathrm{vppm}$ & Ozone & $0.4 \mathrm{vppm}$ \\
helium & $5.2 \mathrm{vppm}$ & Xenon & $0.086 \mathrm{vppm}$ \\
\hline
\end{tabular}

Note: vppm: volume parts per million

From the table, Nitrogen gas $\left(\mathrm{N}_{2}\right)$ represented $78.09 \%$ of air, which was the largest percentage among air components, where Oxygen gas $\left(\mathrm{O}_{2}\right)$ represented $20.95 \%$ of air as the second highest percentage, the third element was Argon gas, which represented $0.93 \%$ of air. These are the main industrial gases produced in large scales for different applications [11].

The Global industrial gas market was worth $\$ 29.2$ billion in 2005 and $\$ 30.9$ billion in 2006, Andrew in 2009 expected demand worth $\$ 40$ billion in 2011 as shown in Fig. 1, this increment in the demand for industrial gases derived the developments in Air Separation Units for further improvements in production cost, production quality and minimizing the risk in production phase [12].

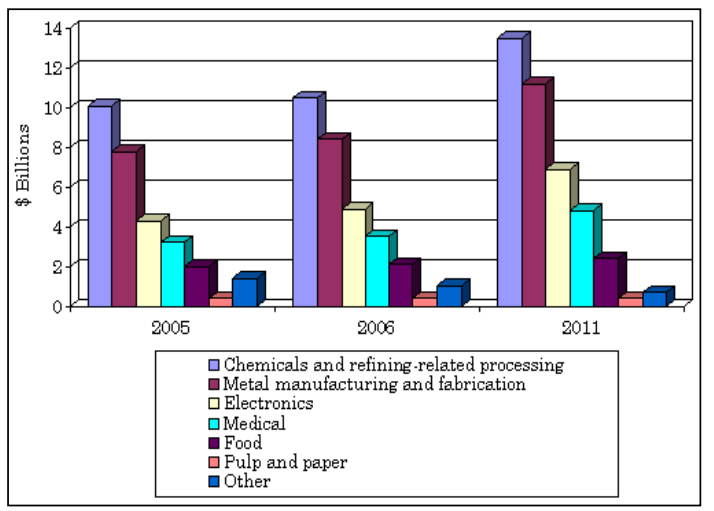

Fig.1. Global industrial gases market value [12].

Air Separation Units consist of five units operation; these are (see Fig. 2): 
- Feed Air Compression unit.

- Feed Air Pre-Treatment unit (purification unit).

- Heat exchange and Liquefaction unit.

- Cryogenic separation unit.

- Product compression unit.

The air compression unit is located at the front end of this process, followed by air treatment unit, which is considered as the safe guard of the plant from the risk of hydrocarbons and water trace. Heat exchange and liquefaction is the core of ASU, where the heat of the feed air is transferred to liquid product and become gases, where the feed itself become liquid air, following to heat exchange step, cryogenic separation, where liquid air distilled to different products as oxygen and nitrogen, which are compressed in the compression unit to meet customer needs [13].

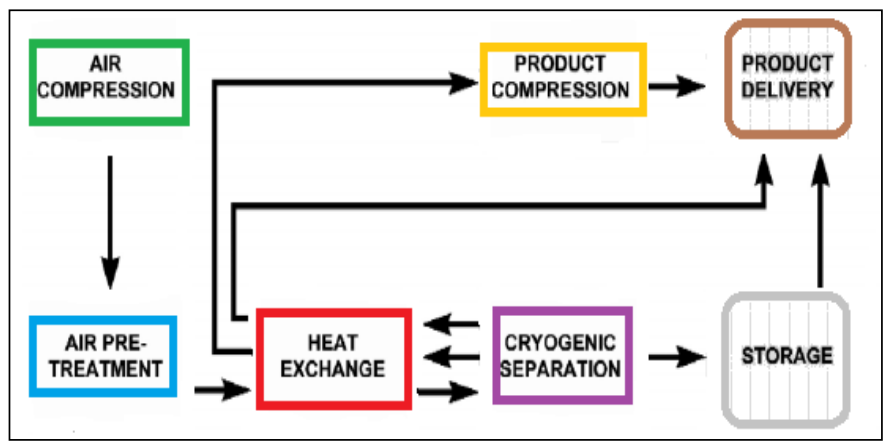

Fig. 2. Units operations for a cryogenic air separation process [13].

The process of industrial gases classified into two classes:

- Cryogenic industrial gas process.

- Non-Cryogenic industrial gas process.

Fig. 3 was sketched to show these classification and the sub technologies in each class [14].

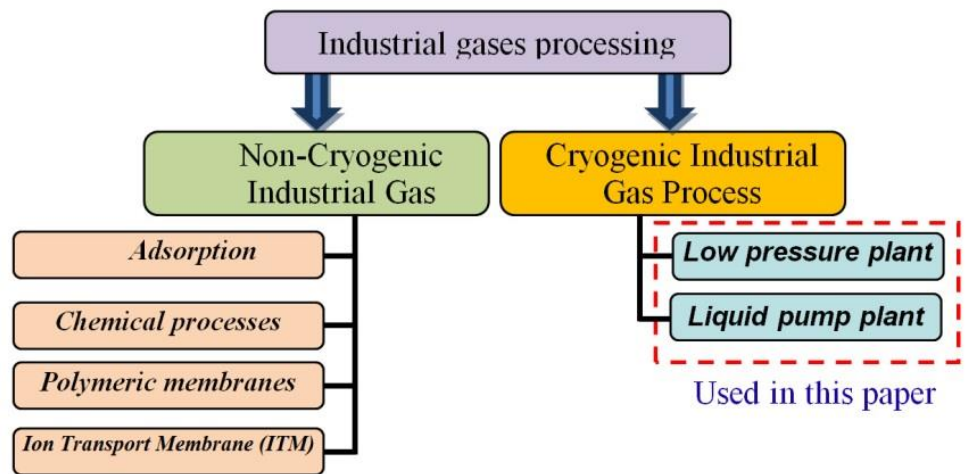

Fig. 3. Classification of industrial gases processing [14].

The Cryogenic industrial gas process consists from two technologies that were used in this paper. These two methods are:

- Low pressure plant with product compressors.

- Liquid pump plant (internal compression pump is used instead of compressors).

The Non-cryogenic industrial gas processes mainly classified into four classes:

- Adsorption.

- Chemical process.

- Polymeric membranes.

- Ion Transport Membrane ( ITM)

The units that are used in this research are;

- Phase three air separation unit (ASU-31). 
- Phase five air separation unit (ASU-51).

- Phase seven air separation unit (ASU-71).

These units equipped with gas purification systems, which had concerned to be very critical area, where the safety and quality started, these areas had been conceded to be area of improvement, where most of power consumption was took place and reflected in overall units performance.

Cryogenic Air Separation Unit (ASU) composed of compressors, heat exchangers, expander and distillation columns where air is separated into nitrogen and oxygen gases by distillation at very low temperatures, the design of cryogenic air separation unites ASU depended on the scale of production and the nature of the products required by customers. While basic principles were always the same, process flows for each plant can vary significantly since ASU's designed to meet specific customer's requirement.

For Low-pressure plant; Air at ambient temperature withdrawn by compressor via intake filter, where dust particles are removed, Compressed air passes to pre-cooling system, which composed of direct contact air cooler, Air passes to purification system composed of Molecular Sieve, Purified air cold down in high efficient heat exchanger, purified air compressed more in booster air compressor then subjected to expansion by turbine, which leads to a significant drop in air temperature to liquefaction point, air gas liquefied at very low temperature less than $-185 \mathrm{C}^{\circ}$, which is the source of the coldness in ASU. Liquid air separated by distillation, where liquid production of oxygen, nitrogen and argon produced. Small amount of liquid product subjected to further cooling in sub-cooler, then transferred to liquid storage tanks as a backup for gas. Finally, these streams of liquid products gain and converted to gas and compressed in order to meet customers' needs.

Other cryogenic ASU technology used internal pump, where liquid oxygen is pumped with elevated pressure matching customer needs before it passed to main heat exchanger for vaporization. This unit normally equipped with nitrogen compressor to elevate nitrogen to customers need.

\section{Key Performance Indicators for Air Separation Unit}

There are 5 mains KPI's that can be used to monitor individual unit operation performance that can be summarized by the followings:

i. Individual air compressors specific powers.

ii. Individual oxygen compressors specific powers.

iii. Individual Nitrogen compressors specific powers.

iv. Molecular Sieve regeneration power.

v. Overall oxygen specific power, which is known as the ratio between the

(Total power consumption in $\mathrm{Kw} / \mathrm{hr}$ ) and (Total oxygen production in $\mathrm{Nm}^{3} / \mathrm{hr}$ ).

The most important parameter of KPI is the oxygen specific power, which is the total power consumption per normal cubic meter of oxygen, this parameter of KPI reflects the overall performance of the ASU, Individual specific power can be used for monitoring individual equipment performance, an example for this, is the air compressor specific power ( $\mathrm{Kw} / \mathrm{Nm}^{3}$ of Air), but this will not reflect the overall ASU performance. Hence, comparison between different ASUs performance normally done by calculating the oxygen specific power since it is involved in the overall production cost.

The design bases for any ASU done according to capacity of oxygen production since oxygen considered as the main product of any ASU. The overall oxygen specific power was selected in this paper as KPI, which will be used as a tool for calculation and comparisons.

\section{Air Separation Unit Theoretical Oxygen Specific Power}

One of the technological forecasting studies done by Castle showed that the oxygen specific power for cryogenic air separation unit will be reduced to about $0.28 \sim 0.3 \mathrm{Kw} / \mathrm{Nm}^{3}$ in year 2010 [11], this technological forecasting was done by extrapolation method as shown in Fig. 4, the forecasted value was confirmed by Pfaff, and Kather, they mentioned that the specific power for up-to dates ASU is approximately $0.25-0.28 \mathrm{Kw} / \mathrm{Nm}^{3}$ [15], which is closed to the earlier forecasting done by Castle [11]. 


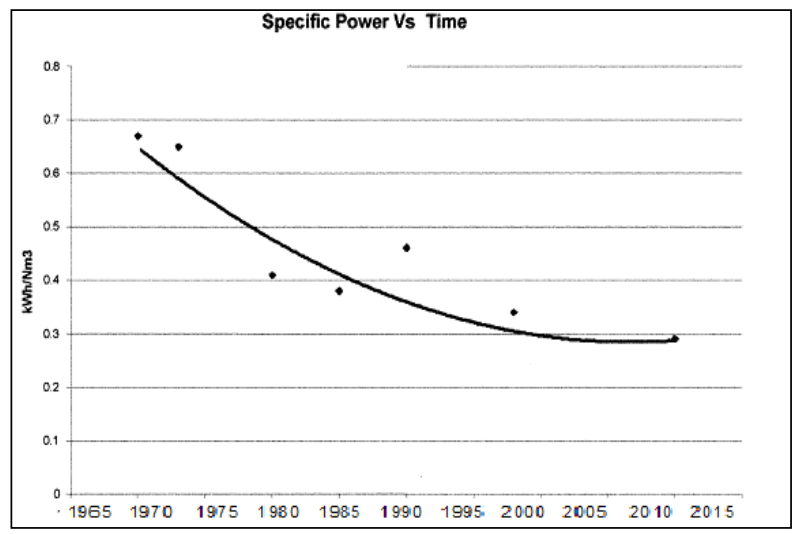

Fig. 4. Specific Power vs. Time [11].

The oxygen specific power value $\left(0.28 \mathrm{Kw} / \mathrm{Nm}^{3}\right)$ will be used as the theoretical benchmark for current air separation used in this paper.

\section{Air Separation Units}

Three air separation units ASU-31, ASU-51 and ASU-71 used to collect data in calculating specific power that was used in comparison in this paper.

\subsection{Air Separation Units ASU-31}

The air separation unit ASU-31 is classified as a low pressure cryogenic plant as shown in Fig. 5, because it is operated with oxygen compressors. The production of this unit is 1200 metric ton/day, which is equivalent to $35000 \mathrm{Nm}^{3} / \mathrm{hr}$. The accuracy of the data collected for oxygen production was not representing the actual production quantity of oxygen gas, the data collected at three places as follows;

- P1: out of the column.

- P2: out of oxygen compressors.

- P3: plant Battery limit (BL).

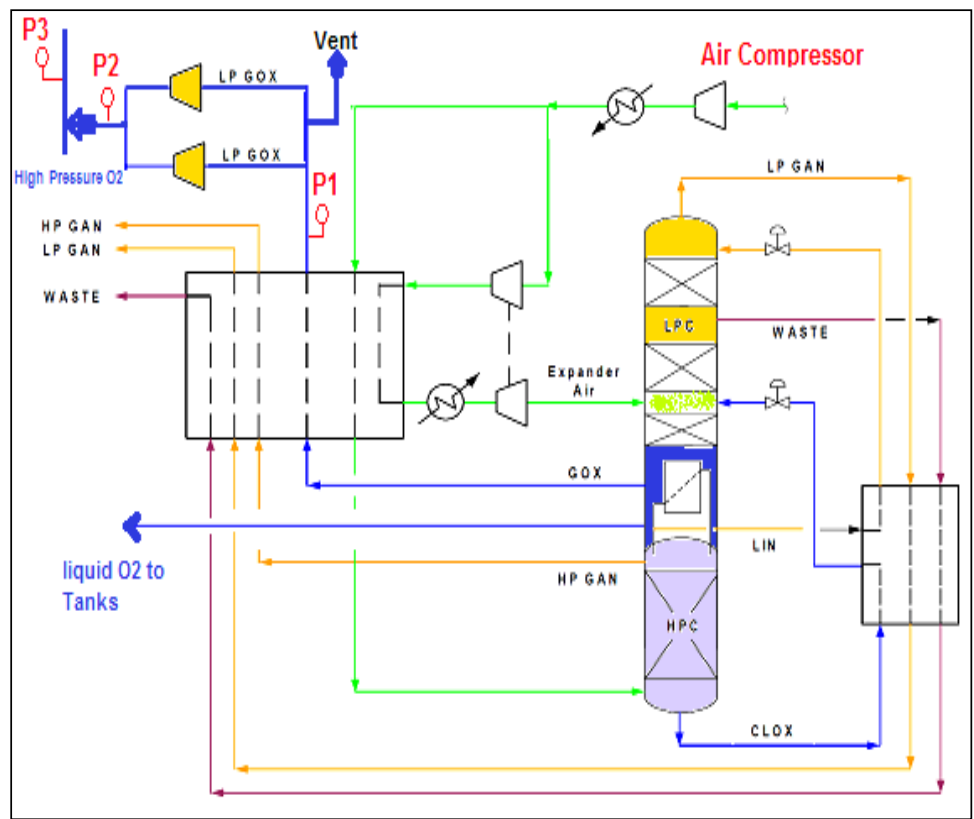

Fig. 5. Locations of $\mathrm{O}_{2}$ production readings in ASU-31.

Note: $\quad L P G O X$ : Low Pressure Gas OXygen

HP GOX: High Pressure Gas Oxygen

HP GAN: High Pressure GAs Nitrogen 


\section{LP GAN: Low Pressure GAs Nitrogen \\ CLOX: Circulating Liquid OXygen \\ LIN: LIquid Nitrogen}

Table 2 shows the gas oxygen production from the three places as P1, P2 and P3. The oxygen specific power was calculated by the following formula [11];

$\mathrm{O}_{2}$ Specific Power in $\left(\mathrm{Kw} / \mathrm{Nm}^{3}\right)=$ Total Power consumption in $(\mathrm{Kw} / \mathrm{hr}) /$ Total $\mathrm{O}_{2}$ production in $\left(\mathrm{Nm}^{3} / \mathrm{hr}\right)$

The oxygen specific power at (P1) represented all the production of oxygen out of the coldbox, where the oxygen specific power at (P2) represented the actual oxygen production compressed to the customers, (P1) did not effected when some of oxygen production vented to the atmosphere, where one compressor was in use. This happened when there was less demand, and this unit did not have the capability to produce the exact quantity of production that must be depended on demand.

Table 2. Power consumption, oxygen production and specific power for ASU-31.

\begin{tabular}{|c|c|c|c|c|c|c|c|c|}
\hline No. & DATE & $\begin{array}{c}\text { Power } \\
\text { Consumption } \\
(\mathrm{Kw} / \mathrm{hr})\end{array}$ & $\begin{array}{c}\text { P1 } \\
\text { Total } \mathrm{O}_{2} \text { Production } \\
\text { at Coldbox Outlet } \\
(\mathrm{Nm} 3 / \mathrm{hr})\end{array}$ & $\begin{array}{c}\text { P2 } \\
\text { Total } \mathrm{O}_{2} \text { Production } \\
\text { out of Compressors } \\
(\mathrm{Nm} 3 / \mathrm{hr})\end{array}$ & $\begin{array}{c}\text { P3 } \\
\text { Total } \mathrm{O}_{2} \text { Production } \\
\text { at Battery Limit } \\
(\mathrm{Nm} 3 / \mathrm{hr})\end{array}$ & $\begin{array}{c}\text { P1 } \\
\mathrm{O}_{2} \text { Specific Power } \\
(\mathrm{Kw} / \mathrm{Nm} 3) \text { at Cold } \\
\text { box }\end{array}$ & \begin{tabular}{|c|} 
P2 \\
$\mathrm{O}_{2}$ Specific Power \\
$(\mathrm{Kw} / \mathrm{Nm} 3)$ at \\
Compressors
\end{tabular} & $\begin{array}{c}\text { P3 } \\
\mathrm{O}_{2} \text { Specific } \\
\text { Power } \\
(\mathrm{Kw} / \mathrm{Nm} 3) \text { at BL }\end{array}$ \\
\hline 1. & $08 / 09 / 2009$ & 18386 & 35372 & 35790 & 25912 & 0.520 & $0.514^{5}$ & $0.710^{2}$ \\
\hline 2. & 09/09/2009 & 18424 & 35138 & 8666 & 19444 & 0.524 & $2.126^{1}$ & $0.948^{2}$ \\
\hline 3. & $10 / 09 / 2009$ & 18212 & 35004 & 5910 & 20685 & 0.520 & $3.081^{1}$ & $0.880^{2}$ \\
\hline 4. & $11 / 09 / 2009$ & 18091 & 34552 & 30680 & 28754 & 0.524 & 0.590 & 0.629 \\
\hline 5. & $12 / 09 / 2009$ & 18120 & 34597 & 35995 & 29840 & 0.524 & 0.503 & 0.607 \\
\hline 6. & $13 / 09 / 2009$ & 18150 & 34644 & 35058 & 29817 & 0.524 & 0.518 & 0.609 \\
\hline 7. & \begin{tabular}{|l|}
$14 / 09 / 2009$ \\
\end{tabular} & 18189 & 34623 & 34982 & 29754 & 0.525 & 0.520 & 0.611 \\
\hline 8. & $15 / 09 / 2009$ & 18257 & 34786 & 35076 & 29773 & 0.525 & 0.520 & 0.613 \\
\hline 9. & $16 / 09 / 2009$ & 18185 & 34676 & 36013 & 29903 & 0.524 & 0.505 & 0.608 \\
\hline 10. & $17 / 09 / 2009$ & 17925 & 34407 & 36078 & 30042 & 0.521 & 0.497 & 0.597 \\
\hline 11. & \begin{tabular}{|l|}
$18 / 09 / 2009$ \\
\end{tabular} & 17399 & 33644 & 36215 & 29824 & 0.517 & 0.480 & 0.583 \\
\hline 12. & $19 / 09 / 2009$ & 17295 & 33150 & 34766 & 29621 & 0.522 & 0.497 & 0.584 \\
\hline 13. & $20 / 09 / 2009$ & 17411 & 33169 & 35642 & 29210 & 0.525 & 0.489 & 0.596 \\
\hline 14. & $21 / 09 / 2009$ & 17330 & 33159 & 36883 & 29209 & 0.523 & 0.470 & 0.593 \\
\hline 15. & $22 / 09 / 2009$ & 17555 & 33081 & 36742 & 29360 & 0.531 & 0.478 & 0.598 \\
\hline 16. & $23 / 09 / 2009$ & 17376 & 33126 & 36561 & 29421 & 0.525 & 0.475 & 0.591 \\
\hline 17. & $24 / 09 / 2009$ & 17281 & 32888 & 36400 & 29305 & 0.525 & 0.475 & 0.590 \\
\hline 18. & $25 / 09 / 2009$ & 17225 & 32725 & 36504 & 29393 & 0.526 & 0.472 & 0.586 \\
\hline 19. & $26 / 09 / 2009$ & 17210 & 32805 & 36300 & 29445 & 0.525 & 0.474 & 0.584 \\
\hline 20. & $27 / 09 / 2009$ & 17589 & 33518 & 35939 & 29808 & 0.525 & 0.489 & 0.590 \\
\hline 21. & $28 / 09 / 2009$ & 17596 & 33327 & 36015 & 29946 & 0.528 & 0.489 & 0.588 \\
\hline 22. & $29 / 09 / 2009$ & 17625 & 33118 & 36167 & 29971 & 0.532 & 0.487 & 0.588 \\
\hline 23. & $30 / 09 / 2009$ & 17448 & 33037 & 38060 & 29946 & 0.528 & 0.458 & 0.583 \\
\hline 24. & $01 / 10 / 2009$ & 17435 & 33016 & 38267 & 29439 & 0.528 & 0.456 & 0.592 \\
\hline 25. & $02 / 10 / 2009$ & 17315 & 32980 & 36768 & 29442 & 0.525 & 0.471 & 0.588 \\
\hline 26. & $03 / 10 / 2009$ & 14816 & 2367 & 2843 & 28496 & $6.259^{6}$ & $5.211^{1}$ & 0.520 \\
\hline 27. & $04 / 10 / 2009$ & 16553 & 22719 & 7146 & 27606 & 0.729 & $2.316^{1}$ & 0.600 \\
\hline 28. & $05 / 10 / 2009$ & 17870 & 35165 & 4535 & 21614 & 0.508 & $3.941^{1}$ & $0.827^{2}$ \\
\hline 29. & $06 / 10 / 2009$ & 18285 & 35877 & 10257 & 20168 & 0.510 & $1.783^{1}$ & $0.907^{2}$ \\
\hline 30. & $07 / 10 / 2009$ & 18216 & 34829 & 29691 & 19433 & 0.523 & 0.614 & $0.937^{2}$ \\
\hline 31. & 08/10/2009 & 18295 & 34916 & 35971 & 19277 & 0.524 & 0.509 & $0.949^{2}$ \\
\hline 32. & $09 / 10 / 2009$ & 18238 & 35220 & 35838 & 19294 & 0.518 & 0.509 & $0.945^{2}$ \\
\hline 33. & $10 / 10 / 2009$ & 18187 & 35050 & 36185 & 19379 & 0.519 & 0.503 & $0.938^{2}$ \\
\hline 34. & $11 / 10 / 2009$ & 18154 & 35054 & 35569 & 19284 & 0.518 & 0.510 & $0.941^{2}$ \\
\hline 35. & $12 / 10 / 2009$ & 18253 & 35222 & 23291 & 19445 & 0.518 & 0.784 & 0.939 \\
\hline \multicolumn{9}{|c|}{ Unit Shutdown } \\
\hline 36. & $17 / 12 / 2009$ & 17730 & 35424 & 36743 & 28043 & 0.501 & 0.481 & 0.582 \\
\hline 37. & $18 / 12 / 2009$ & 17712 & 35457 & 36599 & 28064 & 0.500 & 0.484 & 0.847 \\
\hline 38. & $19 / 12 / 2009$ & 17808 & 35449 & 36641 & 28124 & 0.502 & 0.489 & $15.534^{2}$ \\
\hline 39. & $20 / 12 / 2009$ & 17712 & 35510 & 36568 & 28023 & 0.499 & 0.485 & $14.406^{2}$ \\
\hline 40. & $21 / 12 / 2009$ & 17681 & 35651 & 34939 & 27243 & 0.496 & 0.506 & $13.945^{2}$ \\
\hline 41. & $22 / 12 / 2009$ & 17585 & 35488 & 34879 & 27352 & 0.496 & 0.503 & $2.728^{2}$ \\
\hline 42. & $23 / 12 / 2009$ & 17326 & 34819 & 35903 & 27682 & 0.498 & 0.481 & 0.913 \\
\hline 43. & $24 / 12 / 2009$ & 17685 & 35060 & 36200 & 28829 & 0.504 & 0.489 & 0.674 \\
\hline 44. & $25 / 12 / 2009$ & 17822 & 35203 & 35800 & 29586 & 0.506 & 0.496 & 0.599 \\
\hline 45. & $26 / 12 / 2009$ & 17771 & 35414 & 36596 & 29612 & 0.502 & 0.485 & 0.592 \\
\hline 46. & $27 / 12 / 2009$ & 17788 & 35185 & 36456 & 30252 & 0.506 & 0.483 & 0.652 \\
\hline 47. & $28 / 12 / 2009$ & 17754 & 35373 & 38046 & 30513 & 0.502 & $0.464^{3}$ & 0.704 \\
\hline 48. & $29 / 12 / 2009$ & 17657 & 34810 & 38286 & 20839 & 0.507 & $0.464^{3}$ & 0.700 \\
\hline 49. & $30 / 12 / 2009$ & 17453 & 34733 & 30035 & 1124 & 0.503 & 0.579 & $15.534^{2}$ \\
\hline 50. & $31 / 12 / 2009$ & 17414 & 34352 & 22839 & 1209 & 0.507 & $0.763^{4}$ & $14.406^{2}$ \\
\hline 51. & $01 / 01 / 2010$ & 17382 & 34351 & 22840 & 1246 & 0.506 & $0.764^{4}$ & $13.945^{2}$ \\
\hline 52. & $02 / 01 / 2010$ & 17586 & 34736 & 33641 & 6446 & 0.506 & 0.525 & $2.728^{2}$ \\
\hline 53. & $03 / 01 / 2010$ & 17797 & 35008 & 38204 & 19499 & 0.508 & 0.466 & 0.913 \\
\hline 54. & $04 / 01 / 2010$ & 17729 & 35131 & 36431 & 26321 & 0.505 & 0.485 & 0.674 \\
\hline 55. & $05 / 01 / 2010$ & 17975 & 35121 & 36442 & 30022 & 0.512 & 0.492 & 0.599 \\
\hline 56. & $06 / 01 / 2010$ & 17812 & 35123 & 36485 & 30111 & 0.507 & 0.490 & 0.592 \\
\hline 57. & $07 / 01 / 2010$ & 17999 & 35123 & 36430 & 27620 & 0.512 & 0.496 & 0.652 \\
\hline 58. & $08 / 01 / 2010$ & 17973 & 35209 & 36457 & 25537 & 0.510 & 0.491 & 0.704 \\
\hline 59. & $09 / 01 / 2010$ & 17968 & 35202 & 36460 & 25686 & 0.510 & 0.490 & 0.700 \\
\hline 60. & $10 / 01 / 2010$ & 17844 & 35053 & 34322 & 25550 & 0.509 & 0.519 & 0.698 \\
\hline 61. & $11 / 01 / 2010$ & 17841 & 35081 & 23064 & 25230 & 0.509 & 0.774 & 0.707 \\
\hline
\end{tabular}




\begin{tabular}{|l|l|c|c|c|c|c|c|c|}
\hline 62. & $12 / 01 / 2010$ & 18169 & 35165 & 20240 & 24987 & 0.517 & 0.899 & 0.727 \\
\hline 63. & $13 / 01 / 2010$ & 17690 & 33371 & 1221 & 24895 & 0.530 & $14.949^{1}$ & 0.711 \\
\hline 64. & $14 / 01 / 2010$ & 17634 & 32958 & 13727 & 24662 & 0.535 & $1.285^{1}$ & 0.715 \\
\hline \multicolumn{2}{|c|}{ Average } & $\mathbf{1 7 7 2 1}$ & $\mathbf{3 3 8 3 6}$ & $\mathbf{3 1 1 7 6}$ & $\mathbf{2 5 2 4 3}$ & $\mathbf{0 . 6 0 8}$ & $\mathbf{0 . 9 9 9}$ & $\mathbf{2 . 0 6 3}$ \\
\hline
\end{tabular}

Note: $\quad$ 'compressors unloaded, no demand

${ }^{2}$ not all product to P3

3 lowest value, maximum production

${ }^{4}$ one compressor was running, less demand

${ }^{5}$ used as an example

${ }^{6}$ up normal

The average of the power consumption is $(17721 \mathrm{Kw} / \mathrm{hr})$ and the average of the total oxygen production at $\mathrm{P} 1=33836 \mathrm{Nm}^{3} / \mathrm{hr}, \mathrm{P} 2=31176 \mathrm{Nm}^{3} / \mathrm{hr}$ and $\mathrm{P} 3=25243 \mathrm{Nm}^{3} / \mathrm{hr}$. The averages of these values showed large deviation between the three averages; P1 $=0.608 \mathrm{Kw} / \mathrm{Nm}^{3}, \mathrm{P} 2=0.999 \mathrm{Kw} / \mathrm{Nm}^{3}$ and $\mathrm{P} 3=2.063 \mathrm{Kw} / \mathrm{Nm}^{3}$. The oxygen specific power calculated from the data of the battery limit P3 was the highest value compared with the results of $\mathrm{O} 2$ specific power at P2 and P1. The reason for that was withdrawing some of oxygen production out of ASU-31 through the tie-in piping located before P3. The oxygen specific power at P1 represents all production of oxygen out of the coldbox, where the oxygen specific power at $\mathrm{P} 2$ represents the actual oxygen production compressed to the customers,

The averages of oxygen production at $\mathrm{P} 1=33836 \mathrm{Nm}^{3} / \mathrm{hr}, \mathrm{P} 2=31176 \mathrm{Nm}^{3} / \mathrm{hr}$, and $\mathrm{P} 3=25243 \mathrm{Nm}^{3} / \mathrm{hr}$, these averages showed that there are approximately average loss of oxygen between (P1 and P3) $=33836$ $25243=8593 \mathrm{Nm}^{3} / \mathrm{hr}$, and for specific power there are an extra loss of specific power between (P1 and P3) $=2.063-0.608=1.455 \mathrm{Kw} / \mathrm{Nm}^{3}$. The losses need to be minimized by adding the capability of controlling the unit to produce gases according to demand to minimize loss of power.

\subsection{Air Separation Units ASU-51}

The air separation unit ASU-51 classified as a cryogenic Liquid pump plant as shown in Fig. 6, because it was equipped with Internal Compression pump (IC-Pump) instead of oxygen compressors, which were used in ASU-31. ASU-51 was designed to produce 2682 metric ton/day of oxygen, which equivalent to $78225 \mathrm{Nm}^{3} / \mathrm{hr}$. Since there was only one measuring point for oxygen gas, data was collected for the total oxygen production by adding both gaseous oxygen at P4 and liquid oxygen. The total power consumption for ASU-51 was collected by adding all the power consumption of equipments, which were involved in oxygen production as follows:

- Power of air compressors.

- Power of regeneration heaters.

- Power of all pumps involved in production.

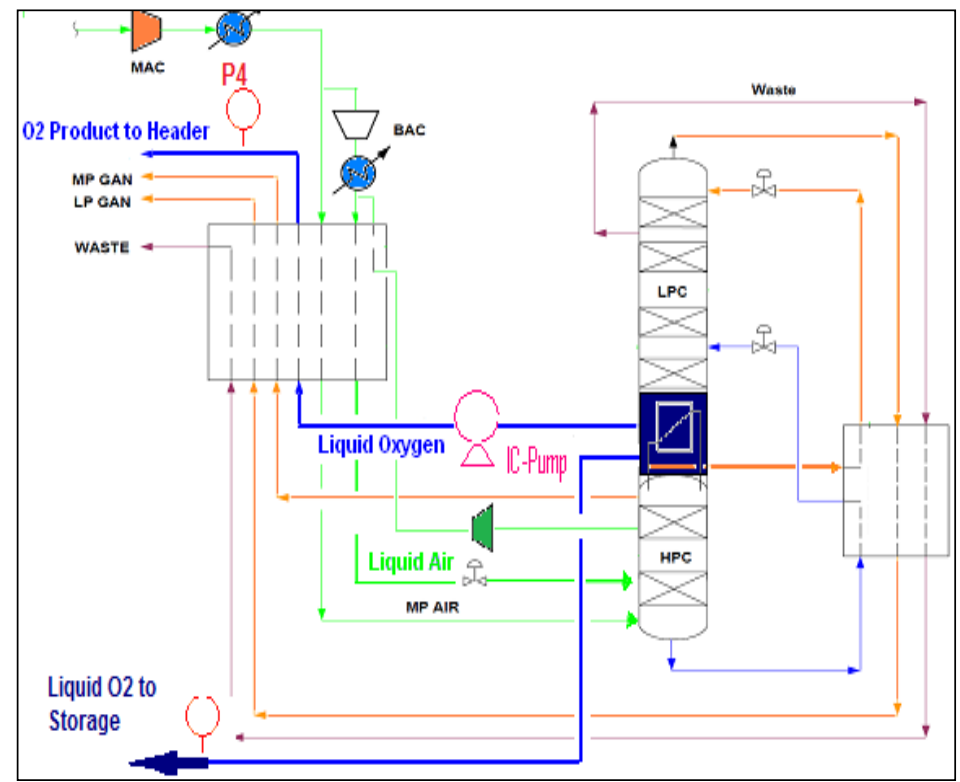

Fig. 6. Location of $\mathrm{O} 2$ production readings in ASU-51. 
Note: $\quad$ LP GOX: Low Pressure OXygen Gas

HP GOX: High Pressure Gas OXygen

MP GAN: Medium Pressure GAs Nitrogen

$L P$ GAN: Low Pressure GAs Nitrogen

MAC: Main Air Compressor

BAC: Booster Air Compressor

IC-Pump: Internal Compression pump

Table 3 shows the total oxygen production and total power consumption.

Table 3. Power consumption, oxygen production and specific power for ASU-51.

\begin{tabular}{|c|c|c|c|c|}
\hline NO. & DATE & $\begin{array}{c}\text { Total Power } \\
\text { Consumption }(\mathrm{Kw} / \mathrm{hr})\end{array}$ & \begin{tabular}{|c|}
$\mathbf{P 4}$ \\
Total $\mathrm{O}_{2}$ Production $\left(\mathrm{Nm}^{3} / \mathrm{hr}\right)$
\end{tabular} & \begin{tabular}{|c|} 
P4 \\
Specific Power $\left(\mathrm{Kw} / \mathrm{Nm}^{3}\right)$
\end{tabular} \\
\hline 1. & \begin{tabular}{|c|}
$8 / 9 / 2009$ \\
\end{tabular} & 48674 & 20652 & 2.357 \\
\hline 2. & 9/9/2009 & 51222 & 76161 & 0.673 \\
\hline 3. & \begin{tabular}{|l|}
$109 / 2009$ \\
\end{tabular} & 50952 & 75285 & 0.677 \\
\hline 4. & \begin{tabular}{|l|}
$11 / 9 / 2009$ \\
\end{tabular} & 50513 & 74861 & 0.675 \\
\hline 5. & \begin{tabular}{|l|}
$12 / 9 / 2009$ \\
\end{tabular} & 50683 & 75193 & 0.674 \\
\hline 6. & \begin{tabular}{|l|}
$13 / 9 / 2009$ \\
\end{tabular} & 50702 & 75294 & 0.673 \\
\hline 7. & \begin{tabular}{|l|}
$14 / 9 / 2009$ \\
\end{tabular} & 50800 & 74936 & 0.678 \\
\hline 8. & \begin{tabular}{|l|}
$15 / 9 / 2009$ \\
\end{tabular} & 50884 & 74450 & 0.683 \\
\hline 9. & \begin{tabular}{|l|}
$16 / 9 / 2009$ \\
\end{tabular} & 50735 & 75058 & 0.676 \\
\hline 10. & \begin{tabular}{|l|}
$17 / 9 / 2009$ \\
\end{tabular} & 50707 & 75012 & 0.676 \\
\hline 11. & \begin{tabular}{|l|}
$18 / 9 / 2009$ \\
\end{tabular} & 50430 & 74230 & 0.679 \\
\hline 12. & \begin{tabular}{|l|}
$19 / 9 / 2009$ \\
\end{tabular} & 49090 & 70886 & 0.693 \\
\hline 13. & $20 / 9 / 2009$ & 48837 & 70445 & 0.693 \\
\hline 14. & \begin{tabular}{|l|}
$21 / 9 / 2009$ \\
\end{tabular} & 48737 & 70451 & 0.692 \\
\hline 15. & $22 / 9 / 2009$ & 48797 & 70466 & 0.692 \\
\hline 16. & \begin{tabular}{|l|}
$23 / 9 / 2009$ \\
\end{tabular} & 48549 & 69946 & 0.694 \\
\hline 17. & $24 / 9 / 2009$ & 48519 & 70130 & 0.692 \\
\hline 18. & \begin{tabular}{|l|}
$25 / 9 / 2009$ \\
\end{tabular} & 48200 & 69494 & 0.694 \\
\hline 19. & $26 / 9 / 2009$ & 47976 & 69419 & 0.691 \\
\hline 20. & \begin{tabular}{|l|}
$27 / 9 / 2009$ \\
\end{tabular} & 48003 & 69948 & 0.686 \\
\hline 21. & $28 / 09 / 2009$ & 48057 & 70426 & 0.682 \\
\hline 22. & $29 / 09 / 2009$ & 47961 & 69928 & 0.686 \\
\hline 23. & $30 / 09 / 2009$ & 48067 & 70054 & 0.686 \\
\hline 24. & $01 / 10 / 2009$ & 48162 & 69305 & 0.695 \\
\hline 25. & $02 / 10 / 2009$ & 48059 & 70164 & 0.685 \\
\hline 26. & $03 / 10 / 2009$ & 49806 & 73180 & 0.681 \\
\hline 27. & $04 / 10 / 2009$ & 50228 & 73703 & 0.681 \\
\hline 28. & 05/10/2009 & 48290 & 69930 & 0.691 \\
\hline 29. & $\mid 06 / 10 / 2009$ & 46138 & 57397 & $0.804^{1}$ \\
\hline 30. & 07/10/2009 & 48751 & 70352 & 0.693 \\
\hline 31. & 08/10/2009 & 50623 & 74578 & 0.679 \\
\hline 32. & 09/10/2009 & 51734 & 77313 & 0.669 \\
\hline 33. & $10 / 10 / 2009$ & 52897 & 79453 & 0.666 \\
\hline 34. & $\mid 11 / 10 / 2009$ & 53017 & 79787 & 0.664 \\
\hline 35. & $12 / 10 / 2009$ & 53046 & 79829 & 0.664 \\
\hline 36. & $13 / 10 / 2009$ & 53034 & 79595 & 0.666 \\
\hline 37. & $14 / 10 / 2009$ & 52938 & 79315 & 0.667 \\
\hline 38. & $\mid 15 / 10 / 2009$ & 52995 & 79107 & 0.670 \\
\hline 39. & $16 / 10 / 2009$ & 51921 & 77472 & 0.670 \\
\hline 40. & $17 / 10 / 2009$ & 47081 & 67480 & 0.698 \\
\hline 41. & $18 / 10 / 2009$ & 47030 & 68331 & 0.688 \\
\hline 42. & $19 / 10 / 2009$ & 47152 & 68617 & 0.687 \\
\hline 43. & $20 / 10 / 2009$ & 47443 & 68707 & 0.691 \\
\hline 44. & $21 / 10 / 2009$ & 47082 & 68188 & 0.690 \\
\hline 45. & $22 / 10 / 2009$ & 46880 & 67972 & 0.690 \\
\hline 46. & $23 / 10 / 2009$ & 46977 & 67721 & 0.694 \\
\hline 47. & $24 / 10 / 2009$ & 47005 & 67718 & 0.694 \\
\hline 48. & $25 / 10 / 2009$ & 47075 & 67987 & 0.692 \\
\hline 49. & $26 / 10 / 2009$ & 47051 & 67398 & 0.698 \\
\hline 50. & $27 / 10 / 2009$ & 47120 & 67097 & 0.702 (Highest Value) \\
\hline 51. & $28 / 10 / 2009$ & 47020 & 67836 & 0.693 \\
\hline 52. & $29 / 10 / 2009$ & 46839 & 68490 & 0.684 \\
\hline 53. & $30 / 10 / 2009$ & 46716 & 67948 & 0.688 \\
\hline 54. & $31 / 10 / 2009$ & 47218 & 67861 & 0.696 \\
\hline 55. & $01 / 11 / 2009$ & 50941 & 74635 & 0.683 \\
\hline 56. & $02 / 11 / 2009$ & 54025 & 79843 & 0.677 \\
\hline 57. & $03 / 11 / 2009$ & 52920 & 77583 & 0.682 \\
\hline 58. & 04/11/2009 & 51990 & 77137 & 0.674 \\
\hline 59. & $05 / 11 / 2009$ & 53949 & 80613 & 0.669 \\
\hline 60. & $06 / 11 / 2009$ & 53807 & 80509 & 0.668 \\
\hline 61. & $07 / 11 / 2009$ & 53762 & 80691 & 0.666 \\
\hline 62. & $08 / 11 / 2009$ & 53774 & 80584 & 0.667 \\
\hline 63. & 09/11/2009 & 53653 & 80810 & 0.664 \\
\hline 64. & $10 / 11 / 2009$ & 53574 & 80674 & 0.664 \\
\hline 65. & $11 / 11 / 2009$ & 51549 & 76928 & 0.670 \\
\hline 66. & $12 / 11 / 2009$ & 51419 & 76703 & 0.670 \\
\hline 67. & $13 / 11 / 2009$ & 51189 & 76696 & 0.667 \\
\hline 68. & $14 / 11 / 2009$ & 50277 & 75030 & 0.670 \\
\hline 69. & $15 / 11 / 2009$ & 48894 & 72139 & 0.678 \\
\hline 70. & $16 / 11 / 2009$ & 53552 & 80836 & 0.662 \\
\hline 71. & $17 / 11 / 2009$ & 53776 & 81208 & 0.662 \\
\hline 72. & $18 / 11 / 2009$ & 53838 & 81901 & 0.657 \\
\hline
\end{tabular}


Note: ${ }^{1}$ unit upset.

\begin{tabular}{|c|c|c|c|c|}
\hline 73. & $19 / 11 / 2009$ & 53277 & 80902 & 0.659 \\
\hline 74. & $20 / 11 / 2009$ & 47912 & 69666 & 0.688 \\
\hline 75. & $\mid 21 / 11 / 2009$ & 20697 & 19904 & $1.040^{1}$ \\
\hline 76. & $22 / 11 / 2009$ & 35290 & 9238 & $3.820^{1}$ \\
\hline 77. & $23 / 11 / 2009$ & 46484 & 64280 & $0.723^{1}$ \\
\hline 78. & $24 / 11 / 2009$ & 46444 & 68695 & 0.676 \\
\hline 79. & $25 / 11 / 2009$ & 47526 & 70751 & 0.672 \\
\hline 80. & $26 / 11 / 2009$ & 48897 & 72713 & 0.672 \\
\hline 81. & $27 / 11 / 2009$ & 53100 & 80673 & 0.658 \\
\hline 82. & $28 / 11 / 2009$ & 53695 & 81008 & 0.663 \\
\hline 83. & $29 / 11 / 2009$ & 53389 & 81129 & 0.658 \\
\hline 84. & $30 / 11 / 2009$ & 53040 & 81049 & 0.654 \\
\hline 85. & $01 / 12 / 2009$ & 53129 & 81136 & 0.655 \\
\hline 86. & $02 / 12 / 2009$ & 52977 & 81246 & 0.652 \\
\hline 87. & $03 / 12 / 2009$ & 53081 & 81322 & 0.653 \\
\hline 88. & $04 / 12 / 2009$ & 53279 & 81401 & 0.655 \\
\hline 89. & $05 / 12 / 2009$ & 53235 & 81959 & 0.650 \\
\hline 90. & |06/12/2009 & 53700 & 83169 & 0.646 \\
\hline 91. & $07 / 12 / 2009$ & 53870 & 83169 & 0.648 \\
\hline 92. & $08 / 12 / 2009$ & 53583 & 83292 & 0.643 \\
\hline 93. & $09 / 12 / 2009$ & 53607 & 83221 & 0.644 \\
\hline 94. & $10 / 12 / 2009$ & 53567 & 83266 & 0.643 \\
\hline 95. & $11 / 12 / 2009$ & 53202 & 83294 & 0.639 (Lowest Value) \\
\hline 96. & $12 / 12 / 2009$ & 53465 & 82927 & 0.645 \\
\hline 97. & $13 / 12 / 2009$ & 52893 & 81923 & 0.646 \\
\hline 98. & $14 / 12 / 2009$ & 48943 & 73118 & 0.669 \\
\hline 99. & $15 / 12 / 2009$ & 50805 & 75349 & 0.674 \\
\hline 100. & $16 / 12 / 2009$ & 52074 & 78666 & 0.662 \\
\hline 101. & 17/12/2009 & 53207 & 80731 & 0.659 \\
\hline 102. & $18 / 12 / 2009$ & 53105 & 80568 & 0.659 \\
\hline 103. & 19/12/2009 & 53578 & 81354 & 0.659 \\
\hline 104. & $20 / 12 / 2009$ & 53312 & 81282 & 0.656 \\
\hline 105. & $21 / 12 / 2009$ & 53140 & 81567 & 0.651 \\
\hline 106. & $22 / 12 / 2009$ & 53136 & 81499 & 0.652 \\
\hline 107. & $23 / 12 / 2009$ & 53436 & 81511 & 0.656 \\
\hline 108. & $24 / 12 / 2009$ & 53546 & 81300 & 0.659 \\
\hline 109. & $25 / 12 / 2009$ & 54706 & 83278 & 0.657 \\
\hline \multicolumn{2}{|c|}{ Average } & 50345 & 73841 & $0.772(\mathrm{Kw} / \mathrm{Nm3})$ \\
\hline
\end{tabular}

The average of the total power consumption is $(50345 \mathrm{Kw} / \mathrm{hr})$ and the average of the total oxygen production at $\mathrm{P} 4$ is $\left(73841 \mathrm{Nm}^{3} / \mathrm{hr}\right)$, this production near to the designed production value of $\left(78225 \mathrm{Nm}^{3} / \mathrm{hr}\right)$, which means the production, was $5.6 \%$ less than the designed production.

The oxygen specific power of air separation of this unit was ranged between $0.639 \mathrm{Kw} / \mathrm{Nm}^{3}$ and $0.702 \mathrm{Kw} / \mathrm{Nm}^{3}$, where the total oxygen production was ranged between $67096 \mathrm{Nm}^{3} / \mathrm{hr}$ and $83293 \mathrm{Nm}^{3} / \mathrm{hr}$. The maximum oxygen specific power was $0.702 \mathrm{Kw} / \mathrm{Nm}^{3}$ during plant turndown mode (less demand by customer).

\subsection{Air Separation Unit ASU-71}

This air separation unit was designed to produce 3000 metric ton/day of oxygen which equivalent to $87500 \mathrm{Nm}^{3} / \mathrm{hr}$. It is operated with Internal Compression pump (IC-Pump), this unit was classified as liquid pump plant, where IC-pump is used instead of oxygen compressors as shown in Fig. 7.

Table 4. Power consumption, oxygen production and specific power for ASU-71.

\begin{tabular}{|l|c|c|c|c|}
\hline NO. & DATE & $\begin{array}{c}\text { Total Power } \\
\text { Consumption }(\mathrm{Kw} / \mathrm{hr})\end{array}$ & $\begin{array}{c}\text { P5 } \\
\text { Total } \mathrm{O}_{2} \text { Production }\left(\mathrm{Nm}^{3} / \mathrm{hr}\right)\end{array}$ & $\begin{array}{c}\text { P5 } \\
\text { Specific Power }\left(\mathrm{Kw} / \mathrm{Nm}^{3}\right)\end{array}$ \\
\hline 1. & $8 / 9 / 2009$ & 61553.48 & 97979 & 0.628 \\
\hline 2. & $9 / 9 / 2009$ & 48503.48 & 73285 & 0.662 \\
\hline 3. & $109 / 2009$ & 48494.05 & 74515 & 0.651 \\
\hline 4. & $11 / 9 / 2009$ & 52888.52 & 83425 & 0.634 \\
\hline 5. & $12 / 9 / 2009$ & 49559.92 & 77507 & 0.639 \\
\hline 6. & $13 / 9 / 2009$ & 49828.07 & 78086 & 0.638 \\
\hline 7. & $14 / 9 / 2009$ & 49085.51 & 76435 & 0.642 \\
\hline 8. & $15 / 9 / 2009$ & 49906.02 & 77426 & 0.645 \\
\hline 9. & $16 / 9 / 2009$ & 49596.62 & 77436 & 0.640 \\
\hline 10. & $17 / 9 / 2009$ & 49871.22 & 78646 & 0.634 \\
\hline 11. & $18 / 9 / 2009$ & 50427.75 & 78499 & 0.642 \\
\hline 12. & $19 / 9 / 2009$ & 49552.70 & 72318 & 0.685 \\
\hline 13. & $20 / 9 / 2009$ & 49007.01 & 71933 & 0.681 \\
\hline 14. & $21 / 9 / 2009$ & 48446.87 & 71655 & 0.676 \\
\hline 15. & $22 / 9 / 2009$ & 48033.62 & 69840 & 0.688 \\
\hline 16. & $23 / 9 / 2009$ & 48987.58 & 73070 & 0.670 \\
\hline 17. & $24 / 9 / 2009$ & 49795.11 & 74968 & 0.664 \\
\hline 18. & $25 / 9 / 2009$ & 49033.87 & 74192 & 0.661 \\
\hline 19. & $26 / 9 / 2009$ & 50835.77 & 77045 & 0.660 \\
\hline 20. & $27 / 9 / 2009$ & 49581.63 & 74474 & 0.666 \\
\hline 21. & $28 / 09 / 2009$ & 48965.46 & 73722 & 0.664 \\
\hline 22. & $29 / 09 / 2009$ & 48944.99 & 73047 & 0.670 \\
\hline 23. & $30 / 09 / 2009$ & 49170.98 & 74207 & 0.663 \\
\hline 24. & $01 / 10 / 2009$ & 49171.73 & 73697 & 0.667 \\
\hline
\end{tabular}




\begin{tabular}{|c|c|c|c|c|}
\hline 25. & $\mid 02 / 10 / 2009$ & 49061.09 & 73818 & 0.665 \\
\hline 26. & $\mid 03 / 10 / 2009$ & 52013.87 & 79180 & 0.657 \\
\hline 27. & $\mid 04 / 10 / 2009$ & 51486.85 & 77954 & 0.660 \\
\hline 28. & $\mid 05 / 10 / 2009$ & 47823.42 & 69903 & 0.684 \\
\hline 29. & |06/10/2009 & 49074.80 & 71365 & 0.688 \\
\hline 30. & 07/10/2009 & 50959.87 & 74416 & 0.685 \\
\hline 31. & 08/10/2009 & 53414.57 & 81632 & 0.654 \\
\hline 32. & 09/10/2009 & 55579.89 & 84251 & 0.660 \\
\hline 33. & $10 / 10 / 2009$ & 55036.42 & 83255 & 0.661 \\
\hline 34. & $\mid 11 / 10 / 2009$ & 55366.59 & 82917 & 0.668 \\
\hline 35. & $12 / 10 / 2009$ & 55594.15 & 83561 & 0.665 \\
\hline 36. & $13 / 10 / 2009$ & 55597.73 & 83462 & 0.666 \\
\hline 37. & $14 / 10 / 2009$ & 54565.96 & 82505 & 0.661 \\
\hline 38. & $15 / 10 / 2009$ & 53336.40 & 79810 & 0.668 \\
\hline 39. & $16 / 10 / 2009$ & 51569.68 & 76159 & 0.677 \\
\hline 40. & $17 / 10 / 2009$ & 48586.53 & 72446 & 0.671 \\
\hline 41. & $\mid 18 / 10 / 2009$ & 47836.63 & 70208 & 0.681 \\
\hline 42. & $19 / 10 / 2009$ & 47894.30 & 70042 & 0.684 \\
\hline 43. & $20 / 10 / 2009$ & 47753.41 & 70146 & 0.681 \\
\hline 44. & $21 / 10 / 2009$ & 47483.85 & 68765 & 0.691 \\
\hline 45. & $22 / 10 / 2009$ & 48385.68 & 72356 & 0.669 \\
\hline 46. & $23 / 10 / 2009$ & 48124.81 & 71478 & 0.673 \\
\hline 47. & $24 / 10 / 2009$ & 47968.27 & 70784 & 0.678 \\
\hline 48. & $25 / 10 / 2009$ & 47104.68 & 67703 & 0.696 \\
\hline 49. & $26 / 10 / 2009$ & 47280.46 & 68045 & 0.695 \\
\hline 50. & $27 / 10 / 2009$ & 47621.45 & 68226 & 0.698 (highest value) \\
\hline 51. & $28 / 10 / 2009$ & 47469.86 & 68813 & 0.690 \\
\hline 52. & $29 / 10 / 2009$ & 47840.63 & 70676 & 0.677 \\
\hline 53. & $30 / 10 / 2009$ & $\begin{array}{l}47457.07 \\
\end{array}$ & 69757 & 0.680 \\
\hline 54. & $\mid 31 / 10 / 2009$ & 50120.48 & 74175 & 0.676 \\
\hline 55. & $\mid 01 / 11 / 2009$ & 56709.54 & 85259 & 0.665 \\
\hline 56. & $02 / 11 / 2009$ & 59618.29 & 90636 & 0.658 \\
\hline 57. & $03 / 11 / 2009$ & 60649.04 & 93351 & 0.650 \\
\hline 58. & $04 / 11 / 2009$ & $\begin{array}{l}61278.64 \\
\end{array}$ & 95473 & 0.642 \\
\hline 59. & $05 / 11 / 2009$ & $\begin{array}{l}60377.52 \\
\end{array}$ & 93675 & 0.645 \\
\hline 60. & $06 / 11 / 2009$ & 59372.13 & 91086 & 0.652 \\
\hline 61. & 07/11/2009 & 54401.71 & 79118 & 0.688 \\
\hline 62. & $08 / 11 / 2009$ & 50566.41 & 75300 & 0.672 \\
\hline 63. & $09 / 11 / 2009$ & 60688.41 & 94554 & 0.642 \\
\hline 64. & $\mid 10 / 11 / 2009$ & 54822.22 & 83555 & 0.656 \\
\hline 65. & $11 / 11 / 2009$ & 48713.58 & 73441 & 0.663 \\
\hline 66. & $12 / 11 / 2009$ & 51119.94 & 78079 & 0.655 \\
\hline 67. & $13 / 11 / 2009$ & 50827.04 & 77151 & 0.659 \\
\hline 68. & $14 / 11 / 2009$ & 48732.20 & 73885 & 0.660 \\
\hline 69. & $15 / 11 / 2009$ & 48998.62 & 73201 & 0.669 \\
\hline 70. & $16 / 11 / 2009$ & 60752.65 & 94099 & 0.646 \\
\hline 71. & $\mid 17 / 11 / 2009$ & 57690.81 & 87672 & 0.658 \\
\hline 72. & $18 / 11 / 2009$ & 51762.23 & 76864 & 0.673 \\
\hline 73. & $19 / 11 / 2009$ & 49401.73 & 73108 & 0.676 \\
\hline 74. & $20 / 11 / 2009$ & 48009.40 & 72535 & 0.662 \\
\hline 75. & $21 / 11 / 2009$ & 55327.40 & 82021 & 0.675 \\
\hline 76. & $22 / 11 / 2009$ & 59999.95 & 89358 & 0.671 \\
\hline 77. & $23 / 11 / 2009$ & 48191.85 & 69838 & 0.690 \\
\hline 78. & $24 / 11 / 2009$ & 47762.22 & 69583 & 0.686 \\
\hline 79. & $25 / 11 / 2009$ & 47845.47 & 69841 & 0.685 \\
\hline 80. & $26 / 11 / 2009$ & 52256.61 & 76383 & 0.684 \\
\hline 81. & $27 / 11 / 2009$ & 58310.56 & 88397 & 0.660 \\
\hline 82. & $28 / 11 / 2009$ & 58571.63 & 87884 & 0.666 \\
\hline 83. & $29 / 11 / 2009$ & 59032.65 & 88778 & 0.665 \\
\hline 84. & $30 / 11 / 2009$ & 59029.89 & 89146 & 0.662 \\
\hline 85. & $\mid 01 / 12 / 2009$ & 59471.78 & 90706 & 0.656 \\
\hline 86. & $\mid 02 / 12 / 2009$ & 59514.50 & 90662 & 0.656 \\
\hline 87. & $03 / 12 / 2009$ & 59407.32 & 90642 & 0.655 \\
\hline 88. & $04 / 12 / 2009$ & 58678.67 & 89242 & 0.658 \\
\hline 89. & $05 / 12 / 2009$ & 59329.67 & 92197 & 0.644 \\
\hline 90. & $06 / 12 / 2009$ & 59464.45 & 92195 & 0.645 \\
\hline 91. & 07/12/2009 & 59871.93 & 93330 & 0.642 \\
\hline 92. & $08 / 12 / 2009$ & 59661.31 & 93161 & 0.640 \\
\hline 93. & $\mid 09 / 12 / 2009$ & 59557.14 & 93367 & 0.638 \\
\hline 94. & $10 / 12 / 2009$ & 59115.09 & 92736 & 0.637 \\
\hline 95. & $11 / 12 / 2009$ & 58287.61 & 90291 & 0.646 \\
\hline 96. & $12 / 12 / 2009$ & 52041.38 & 77694 & 0.670 \\
\hline 97. & $13 / 12 / 2009$ & 53082.93 & 80928 & 0.656 \\
\hline 98. & $14 / 12 / 2009$ & 51241.40 & 77946 & 0.657 \\
\hline 99. & $15 / 12 / 2009$ & 48201.86 & 71663 & 0.673 \\
\hline 100. & $16 / 12 / 2009$ & 54248.18 & 81723 & 0.664 \\
\hline 101. & $17 / 12 / 2009$ & 58847.59 & 87622 & 0.672 \\
\hline 102. & $18 / 12 / 2009$ & 59383.57 & 89458 & 0.664 \\
\hline 103. & $19 / 12 / 2009$ & 60351.45 & 93656 & 0.644 \\
\hline 104. & $20 / 12 / 2009$ & 60238.71 & 94520 & 0.637 \\
\hline 105. & $21 / 12 / 2009$ & 60290.36 & 94549 & 0.638 \\
\hline 106. & $22 / 12 / 2009$ & 60792.06 & 93984 & 0.647 \\
\hline 107. & $23 / 12 / 2009$ & 60049.63 & 94565 & 0.635 \\
\hline 108. & $24 / 12 / 2009$ & 60192.81 & 95412 & 0.631 (lowest value) \\
\hline 109. & $25 / 12 / 2009$ & 60147.39 & 95053 & 0.633 \\
\hline \multicolumn{2}{|c|}{\begin{tabular}{|l|} 
Average \\
\end{tabular}} & 53220 & 80512 & 0.662 \\
\hline
\end{tabular}

The average of the total power consumption was $53220 \mathrm{Kw} / \mathrm{hr}$ and the average of the total oxygen production at P5 was $80512 \mathrm{Nm} 3 / \mathrm{hr}$, this production near to the designed production value of $87500 \mathrm{Nm}^{3} / \mathrm{hr}$, which means production of this unit, was $7.9 \%$ less than the designed production. The 
oxygen specific power was ranged between $0.631 \mathrm{Kw} / \mathrm{Nm}^{3}$ and $0.698 \mathrm{Kw} / \mathrm{Nm}^{3}$, where the total oxygen production was ranged between $67703 \mathrm{Nm}^{3} / \mathrm{hr}$ and $95412 \mathrm{Nm}^{3} / \mathrm{hr}$.

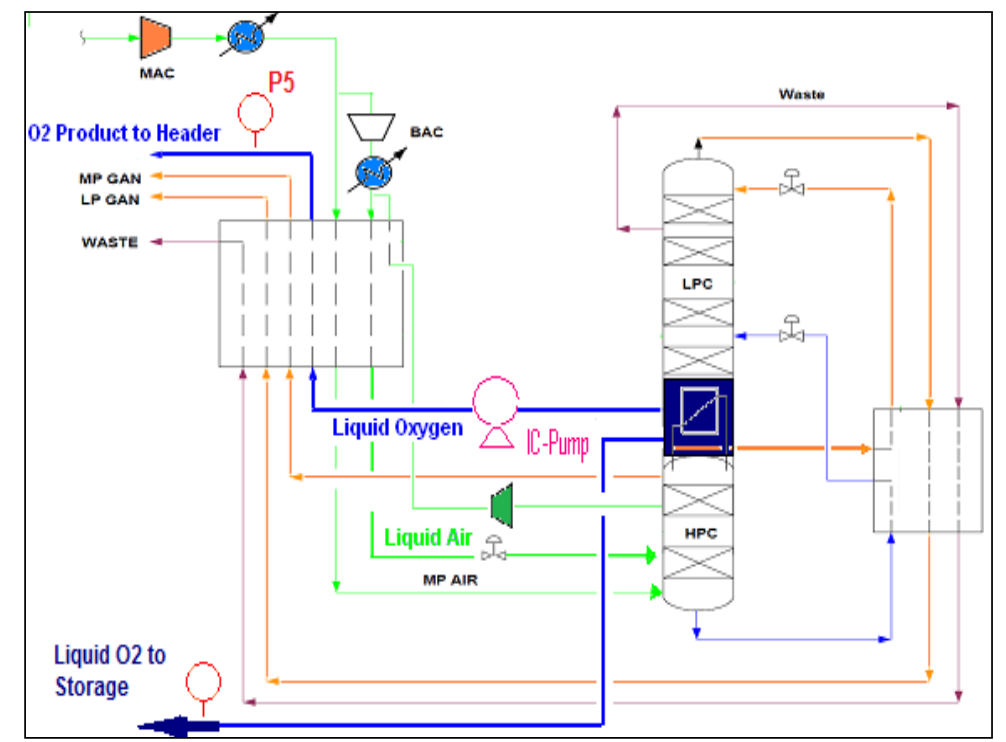

Fig. 7. Locations of $\mathrm{O}_{2}$ production readings in ASU-71.

Note: LP GOX: Low Pressure Gas OXygen

HP GOX: High Pressure Gas Oxygen

HP GAN: High Pressure Gas Nitrogen

LP GAN: Low Pressure Gas Nitrogen

MAC: Main Air Compressor

BAC: Boster Air Compressor

The air separation units ASU-51 and ASU-71 are identical and they have same technology of cryogenic liquid pump plant. Since there was only one measuring point for oxygen gas, data was collected for the total oxygen production by adding both gaseous oxygen and liquid oxygen quantities at P5.

The total power consumption for this unit represented by adding all the power consumption of equipments which were involved in oxygen production as follows;

- Power of air compressors; MAC and BAC.

- Power of regeneration heaters.

- Power of all pumps involved in production.

Table 4 shows the total oxygen production and the total power consumption.

\section{Comparison between Gas Air Separation Units and Benchmark}

The oxygen specific power for ASU-31 was ranged between $0.464 \mathrm{Kw} / \mathrm{Nm}^{3}$ and $0.764 \mathrm{Kw} / \mathrm{Nm}^{3}$ in normal operation with minimum value was $0.464 \mathrm{Kw} / \mathrm{Nm}^{3}$, which will be used as the benchmark of ASU-31. The oxygen specific power for ASU-51 was ranged between $0.639 \mathrm{Kw} / \mathrm{Nm}^{3}$ and $0.702 \mathrm{Kw} / \mathrm{Nm}^{3}$ with average of $0.672 \mathrm{Kw} / \mathrm{Nm}^{3}$, where the minimum value was $0.639 \mathrm{Kw} / \mathrm{Nm}^{3}$, which will be used as the benchmark of ASU-51. The oxygen specific power of ASU-71 was ranged between $0.631 \mathrm{Kw} / \mathrm{Nm}^{3}$ and $0.698 \mathrm{Kw} / \mathrm{Nm}^{3}$ with average of $0.662 \mathrm{Kw} / \mathrm{Nm}^{3}$, with minimum value was $0.631 \mathrm{Kw} / \mathrm{Nm}^{3}$, which will be used as the benchmark of ASU-71. The specific power comparison for ASU-31, ASU-51 and ASU-71 showed a big difference on oxygen specific powers compared with the theoretical benchmark values which is $0.28 \mathrm{Kw} / \mathrm{Nm}^{3}$. Fig. 8 shows the comparison of the three units compared with the benchmark. 


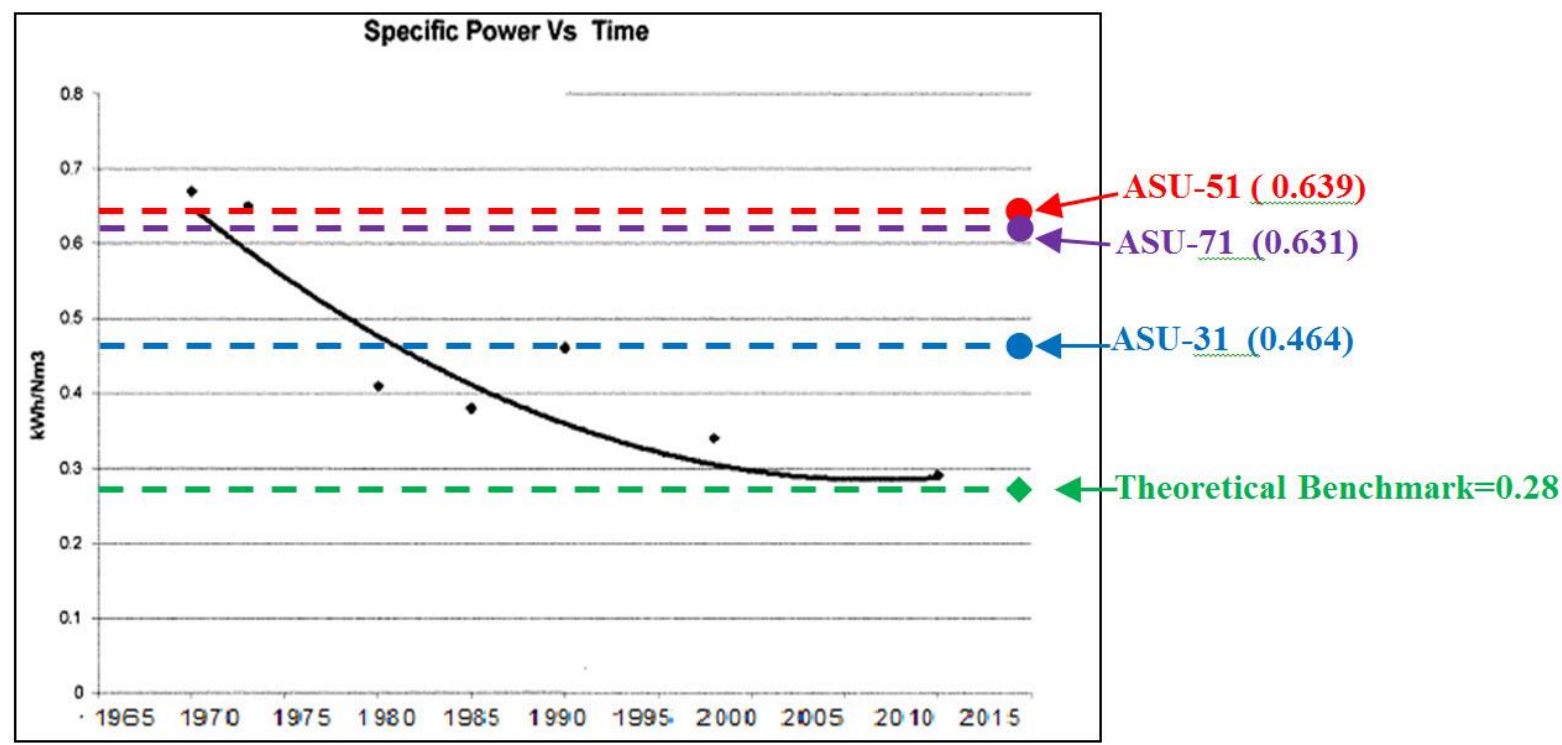

Fig. 8. Benchmarking for O2 specific power of Air Separation Units.

ASU-31 had the lowest oxygen specific power among other operated ASU's, since it was $0.464 \mathrm{Kw} / \mathrm{Nm}^{3}$. The Figure shows also that both ASU-51 and ASU71 with oxygen specific power $0.639 \mathrm{Kw} / \mathrm{Nm}^{3}$ and $0.631 \mathrm{Kw} / \mathrm{Nm}^{3}$ respectively. The air separation units ASU-31 is the most effective unit compared with ASU-51 and ASU71 but still it's specific power is higher than the theoretical benchmark by $0.184 \mathrm{Kw} / \mathrm{Nm}^{3}$, the reason behind this is the difference of technology been used on ASU-31, since it is a low pressure plant ASU technology compared with ASU-51 and ASU-71. The oxygen specific power of ASU-51 was higher than the theoretical benchmark by $0.359 \mathrm{Kw} / \mathrm{Nm}^{3}$, where the oxygen specific power of ASU-71 was higher than the theoretical benchmark by $0.351 \mathrm{Kw} / \mathrm{Nm}^{3}$. ASU-51 performance was very close to ASU-71, these two units have the same technology, which is liquid pump plant. The overall performance showed the needs for adoption of forecasted developments in ASUs units, where power consumption can be reduced in the future.

The ASU-31 is the most cost effective unit in three air separation units since it had the lowest oxygen specific power; this unit was classified as low pressure ASU, where oxygen compressors used in the process, while the other units ASU-51 and ASU-71 are classified as Liquid pump plant, since it was equipped with IC-Pumps.

\section{Conclusion}

Cryogenic air separation units were considered to be the most cost effective technologies used for industrial gases production for large quantities and high purity.

The oxygen specific power comparison between the three units of air separation units ASU-31, ASU51 and ASU-71, showed that ASU-31 is the most cost effective unit. The comparison of the three units of air separation units with the theoretical benchmark of oxygen specific power $(0.28 \mathrm{Kw} / \mathrm{Nm} 3)$ showed there is a difference of $0.184 \mathrm{Kw} / \mathrm{Nm} 3$ for ASU-31, $0.359 \mathrm{Kw} / \mathrm{Nm} 3$ for ASU-51 and $0.351 \mathrm{Kw} / \mathrm{Nm} 3$ for ASU-71. Forecasting of the oxygen specific power can be reduced toward the benchmark which is $(0.28 \sim 0.3$ $\mathrm{Kw} / \mathrm{Nm} 3$ ) using one of the followings suggested technologies;

i. Minimize oxygen losses with nitrogen waste, by increasing the capability of operating the units according to gasses demand.

ii. Adoption of New generation of adsorption beds, that can be regenerated with low temperature less power consumption.

iii. Adoption of the new generation of magnetic bearing, seals if applicable will reduce the frication and eventually reduce the power consumption. 


\section{References}

[1] F. Betz, Managing Technological Innovation, Competitive Advantage from Change. Wiley and Sons Inc, 2003.

[2] V. Garde and R. Patel, "Technological forecasting for power generation - A study using the Delphi Technique," Long Range Planning Journal, vol. 18, pp. 73-79, 1985.

[3] S. Mishra, S. Deshmukh, and P. Vrat, "Technological forecasting applications: Framework and case study on combat vehicles," Defence Science Journal, vol. 53, pp. 371-391, 2003.

[4] P. Pecas, I. Ribeiro, R. Folgado, and E. Henriques, "A life cycle engineering model for technology selection: A case study on plastic injection moulds for low production volumes," Journal of Cleaner Production," vol. 17, pp. 846-856, 2009.

[5] J. Armstrong, "Combining forecasts: The end of the beginning or the beginning of the end?," International Journal of Forecasting, vol. 5, no. 4, pp. 585, 1989.

[6] M. S. Slocum and C. O. Lundberg, "Technology forecasting: From emotional to empirical," Creativity and Innovation Management, vol. 10, no. 2, pp. 139-152, 2001.

[7] J. S. Armstrong and K. C. Green. (2005). Demand Forecasting: Evidence-based Methods. [Online]. Available: http://www.buseco.monash.edu.au/depts/ebs/pubs/wpapers, [Accessed: 20 November 2012].

[8] S. Mishra, S. Deshmukh, and P. Vrat, "Matching of Technological forecasting technique with a technology," Technological Forecasting and Social Change Journal, vol. 69, pp. 1-27, 2002.

[9] A. L. Porter, W. B. Ashton, G. Clar, J. F. Coates, K. Chuls, S. W. Cunningham, K. Ducatel, P. van der Duin, L. Georgehiou, T. Gordon, H. Linstone, V. Marchau, G. Massari, I. Miles, M. Mogee, A. Salo, F. Scapolo, R. Smits, and W. Thissen, "Technology Future Analysis: Toward integration of the field and new methods," Technological Forecasting and Social Change Journal, vol. 71, pp. 287-303, 2004.

[10] A. K. Firat, W. L. Woon, and S. Madnick, "Technological forecasting-A review," Massachusetts Institute of Technology, Cambridge, USA, Working Paper CISL\# 2008-15, 2008.

[11] W. F. Castle, "Air separation and liquefaction: Recent developments and prospects for the beginning of the new millennium," International Journal of Refrigeration, vol. 25, pp. 158-172, 2002.

[12] R. Andrews. (2006). Medtech executives can improve strategic planning with a structured method for exploring emerging technologies. MX Magazine, Foster-Miller Inc. [Online]. Available: http://www.fostermiller.com

[13] A. R. Smith and J. Klosek, "A review of air separation technologies and their integration with energy conversion processes," Fuel Processing Technology, vol. 70, pp. 115-134, 2001.

[14] Y. Alsultanny and N. Al-Shammari, "Oxygen specific power reduction for air separation," Engineering Journal, vol. 17, no. 1, pp. 121-136, 2013.

[15] I. Pfaff and A. Kather, Comparative Thermodynamic analysis and integration issues of CCS steam power plants based on oxy-combustion with cryogenic or membrane based air separation," Energy Procedia Journal, vol. 1, pp. 495-502, 2009. 